\title{
Exon-skipping brain natriuretic peptide variant is overexpressed in failing myocardium and attenuates brain natriuretic peptide production in vitro
}

\author{
Mario Torrado ${ }^{1}$, Raquel Iglesias ${ }^{1}$, Alberto Centeno ${ }^{2}$, Eduardo López ${ }^{2}$, Alexander T \\ Mikhailov $^{1}$ \\ ${ }^{1}$ Developmental Biology Unit, Institute of Health Sciences, University of La Coruña, Campus de Oza, \\ Building 'El Fortin', As Xubias Str. s/n \\ ${ }^{2}$ Experimental Surgery Unit, University Hospital Centre of La Coruña, La Coruña 15006, Spain
}

\begin{abstract}
Brain natriuretic peptide/natriuretic peptide precursor B (NPPB) is one of the most studied genes in relation to heart failure (HF) conditions. However, it is still unclear as to whether alternative splicing could create NPPB mRNA variants, which may be expressed in normal and diseased myocardium. We aimed to identify and characterize a novel alternatively spliced variant of porcine and human NPPB resulting from exon 2 skipping (designated as $\triangle \mathrm{E} 2-\mathrm{NPPB}$ ). A variety of conventional molecular, biochemical and immunochemical methods were used to examine the expression and functional consequences of $\triangle \mathrm{E} 2-\mathrm{NPPB}$ in vitro and in vivo. The pig $\triangle \mathrm{E} 2-\mathrm{NPPB} \mathrm{mRNA}$ is effectively translated into stable protein in cell-based assays but, in contrast to normally spliced NPPB, the $\triangle \mathrm{E} 2-\mathrm{NPPB}$ protein is not secreted into the media. Co-transfection assays demonstrate that $\triangle \mathrm{E} 2-\mathrm{NPPB}$ attenuates production and secretion of normally spliced NPPB, suggesting a negative feedback loop of NPPB signaling through generation of $\triangle \mathrm{E} 2-\mathrm{NPPB}$. The inhibitory effects of $\triangle \mathrm{E} 2-$ NPPB on the expression of NPPB are associated with sequence elements residing in exon 3 of $\triangle \mathrm{E} 2-\mathrm{NPPB}$. In piglets, $\triangle \mathrm{E} 2-\mathrm{NPPB}$ gene expression is downregulated in both ventricles after birth, but it is markedly reactivated in the postnatal myocardium in experimental diastolic heart failure. In addition, we demonstrate that the exon-skipped NPPB variants are expressed in the postnatal and adult human myocardium and upregulated at end-stage HF due to dilated cardiomyopathy. Our work uncovers an important role of alternative exon skipping in the regulation of NPPB gene expression, thereby pinpointing a putative new mechanism for posttranscriptional regulation of NPPB production and secretion.
\end{abstract}

Keywords

NPPB; Exon skipping; Post-transcriptional regulation; Heart failure; Myocardium 


\section{Introduction}

Brain natriuretic peptide (BNP), originally identified in porcine brain, is produced predominantly in the heart as a natriuretic peptide precursor B (NPPB) prepro protein. It is then released as pro-BNP and post-translationally modified by endopeptidase cleavage to form the $\mathrm{N}$ terminal (NT) and biologically active C-terminal (CT) peptide also known as B-type natriuretic peptide, or simply BNP. ${ }^{1}$ Elevated circulating levels of BNP have been found to have prognostic, risk stratification and therapeutic implications in various cardiovascular disorders, especially in systolic $^{2-4}$ and diastolic ${ }^{5-7}$ heart failure (HF).

Genes for mammalian NPPB have been characterized and consist of three exons separated by two introns. ${ }^{8}$ It is believed that most NPPB is synthesized in response to NPPB gene promoter activation by physiological or pathological stimuli. ${ }^{3,4,8,9}$ The main stimulus for NPPB synthesis and secretion from cardiomyocytes is biomechanical stretch resulting from pressure overload, volume expansion or increased filling pressure. ${ }^{5,10,11}$ Since NPPB gene expression was found to be rapidly induced by a variety of cardiac expressed transcriptional factors (including GATA4, NKX2.5 and MEF-2), which are thought to activate NPPB production in myocardium through transcriptional mechanisms, ${ }^{8,9,12}$ possible post-transcriptional control has been largely ignored. However, there is evidence that posttranscriptional regulation can also play a role in both NPPB production and secretion. $^{13,14}$

Alternative splicing is a prevalent mode of posttranscriptional regulation of approximately onehalf of all mammalian genes. Recently, the alternatively spliced NPPB transcript, resulting from intron 2 retention, was identified in failing human left ventricular (LV) myocardium. ${ }^{15} \mathrm{We}$ employed differential mRNA display of the LV versus right ventricular (RV) myocardium in newborn piglets to identify putative marker genes for distinct patterns of postnatal hypertrophic remodeling. One of the transcripts that were predominantly upregulated in the LV revealed a strong sequence homology with both exons 1 and 3 of the $N P P B$ gene. ${ }^{16}$ In the present work, we identify and characterize this transcript as a novel alternatively spliced variant of porcine NPPB resulting from exon 2 skipping (designated as $\triangle \mathrm{E} 2-\mathrm{NPPB})$.

In piglets, $\triangle \mathrm{E} 2-\mathrm{NPPB}$ expression is downregulated in both ventricles shortly after birth, but it is markedly re-activated in myocardium at experimental HF. Our study also provides evidence that $\triangle \mathrm{E} 2-\mathrm{NPPB}$ can post-transcriptionally repress both NPPB production and secretion in cell-based assays, thereby revealing a novel negative feedback mechanism that might influence the production of normally spliced NPPB in experimental HF settings in vivo. In addition, exonskipped NPPB transcripts were detected in normal and failing human myocardium, suggesting the existence of similar mechanisms by which levels of normally spliced NPPB might be regulated in patients.

\section{Materials and methods}

\section{Animals and experimental procedures}

Six-day-old piglets were randomized into two groups (12 animals each), and assigned to receive a single intravenous (i.v.) injection of isotonic saline (phosphate-buffered saline [PBS]) or $1.5-2.0 \mathrm{mg} / \mathrm{kg}$ of doxorubicin (Dox; Sigma, Madrid, Spain) as described in ref. ${ }^{17}$ On day 24 after injection, cardiac output and extravascular lung water were monitored in closed-chest piglets by a PiCCO device (PulsionAG, Munich, Germany) in accordance with the manufacturer's recommendations. The measurements of ventricular end-systolic and end-diastolic pressure were performed in open-chest piglets as described in refs. ${ }^{18,19}$ The piglets were then euthanized to harvest cardiac tissues for RNA isolation. Myocardial samples were also isolated from newborn piglets. Samples were frozen in liquid nitrogen immediately after isolation and then stored until use at $-80^{\circ} \mathrm{C}$. Experimental procedures were carried out in accordance with the European Commission Directive 86/609/EEC on the protection of animals used for experimental and other 
scientific purposes, and all protocols were approved by the Institutional Animal Care and Use Ethical Committee.

\section{Patient cardiac samples}

Samples of non-failing LV myocardium were obtained from five donor hearts not transplanted for technical reasons. Failing myocardial LV samples were obtained from explanted hearts of five patients undergoing orthotropic heart transplantation due to end-stage HF resulting from dilated cardiomyopathy, which was confirmed by clinical examinations. ${ }^{20}$ Cardiac specimens were also collected from one infant patient ( 8 weeks old) with heart septal defects. Samples were collected into RNAlater solution (Ambion, Madrid, Spain), assayed in accordance with the manufacturer's recommendations, and stored at $-80^{\circ} \mathrm{C}$. Informed consent was obtained from patient relatives, and the protocol was approved by the respective Institutional Ethics Committee. The research adheres to the principles outlined in the Declaration of Helsinki.

\section{RNA isolation}

Deep-frozen cardiac tissue samples were directly disrupted in RLT buffer (Qiagen, Madrid, Spain) using a high-speed rotor-stator homogenizer (Ultra-Turrax T8, IKA Labortechnik, Staufen, Germany), digested with Proteinase K (Qiagen), loaded into a RNeasy Midi column (Qiagen), subjected to on-column digestion of DNA with RNase-free DNase (Qiagen) and the protocol was completed in accordance with the manufacturer's recommendations. Total RNA was also isolated from separate batches of transfected COS-7 cells using RNeasy Mini Columns (Qiagen) as just described. Resulting RNA preparations were ethanol-precipitated, re-suspended in RNase-free water and kept at $-80^{\circ} \mathrm{C}$. RNA yield and purity was determined spectrophotometrically at 260-280 $\mathrm{nm}$ and RNA integrity was verified by running samples on $1.5 \%$ agarose gels and staining with ethidium bromide.

\section{Semiquantitative reverse transcription-polymerase chain reaction}

Two micrograms of total RNA were reverse transcribed (RT) using SuperScript III reverse transcriptase (Invitrogen, Barcelona, Spain) and oligo(dT) ${ }^{12-18}$ primer according to the manufacturer's instructions. Piglet and human cardiac cDNAs were used for PCR reactions in a Biometra II PCR system to detect different RNAs using the primers indicated in Table 1. Semiquantitative reverse transcription-polymerase chain reaction (RT-PCR) was performed as described previously. ${ }^{17,20}$ Each RT-PCR setup included primers for the candidate (pig/human NPPB variants) and control (pig/human rpl19 gene coding for ribosomal protein L19). All PCR setups, including no-RT and no template (NT) controls, were performed at least in duplicate. Each PCR sample was resolved on a $2 \%$ agarose gel, and PCR products were visualized with ethidium bromide staining and UV illumination. Band intensity was estimated by densitometry (VersaDoc 1000) and Quantity One software (Bio-Rad, Madrid, Spain). The PCR products were cloned and sequenced (Secugen, Madrid, Spain) to confirm their identity.

\section{Quantitative realtime $R T-P C R$}

Two-step quantitative realtime RT-PCR (qPCR) was performed on a Bio-Rad IQ5 detection system with SYBR Green I mix. ${ }^{19,21}$ The cycling parameters used were standard for SYBR Green I analysis. The reference rpl19 gene was amplified to normalize expression. For each RNA sample, genomic DNA contamination was determined by PCR on a no-RT control for the rpl19 gene. Within each experiment, PCR reactions were done in triplicate. Each PCR reaction was evaluated by melting curve analysis and checking the PCR products on $8 \%$ SYBR Green-stained polyacrylamide gels. Fold changes were calculated using the $\mathrm{C}_{\mathrm{T}}$ method. Data were analyzed using IQ5 optical system software 2.0 and $\mathrm{C}_{\mathrm{T}}$ comparative analysis. For primer sequences used in $\mathrm{qPCR}$ analysis, please see Table 1 . 
Table 1 Primers used in this study

\begin{tabular}{lccccc}
\hline \multirow{2}{*}{ Primer } & Species & Gene & Primer sequence (from 5' to 3') & F/R & Used in \\
\hline & & & & & \\
289 & $\mathrm{p}$ & $n p p b$ & GGC TGC TAG GAA GTG AAA AGT G & $\mathrm{F}$ & RT-PCR \\
82 & $\mathrm{p}$ & $n p p b$ & GCT CCT GCT CCT GTT CTT GCA & $\mathrm{R}$ & qPCR \\
251 & $\mathrm{p}$ & $n p p b$ & GGT CCA GCA GCT CCT GTA TC & $\mathrm{F}$ & RT-PCR, qPCR \\
288 & $\mathrm{p}$ & $n p p b$ & AAG AAC CGT CTT ATA TAC AAC CAC & $\mathrm{F}$ & RT-PCR \\
252 & $\mathrm{p}$ & $n p p b$ & AGT ACC TCC TGA GCA CTG TAT C & $\mathrm{F}$ & RT-PCR, qPCR \\
291 & $\mathrm{~h}$ & $n p p b$ & AAG CAA ACC CGG ACG CAT CG & $\mathrm{R}$ & RT-PCR \\
84 & $\mathrm{~h}$ & $n p p b$ & CTC CTG CTC TTC TTG CAT CTG & $\mathrm{R}$ & RT-PCR \\
286 & $\mathrm{~h}$ & $n p p b$ & ATG TTG ACT TTA TTT CAC CGT GGA & $\mathrm{F}$ & RT-PCR \\
287 & $\mathrm{~h}$ & $n p p b$ & ATG CCG CCT CAG CAC TGT AAC & $\mathrm{F}$ & RT-PCR \\
36 & $\mathrm{p} / \mathrm{h}$ & $r p l 19$ & AAC TCC CGT CAG CAG ATC CG & $\mathrm{R}$ & RT-PCR \\
65 & $\mathrm{p} / \mathrm{h}$ & $r p l 19$ & CTT GGT CTC TTC CTC CTT GGA & $\mathrm{F}$ & RT-PCR \\
64 & $\mathrm{p}$ & $r p l 19$ & CTG CTC AGA AGA TAC CGT GAA T & $\mathrm{R}$ & qPCR \\
206 & $\mathrm{p}$ & $r p l 19$ & GCT TGT GGA TGT GCT CCA TGA & $\mathrm{F}$ & qPCR \\
& & & & & \\
\hline
\end{tabular}

p: pig; h: human; F/R: forward/reverse primers; $n p p b$ : gene coding for natriuretic peptide precursor B preprotein; rpl19: gene coding for ribosomal protein L19; RT-PCR, reverse transcription-polymerase chain reaction; qPCR, quantitative realtime RT-PCR

\section{Gene cloning and expression in Escherichia coli}

Full-length (FL) and partial (exon 3) cDNA sequences of the pig $\triangle E 2-N P P B$, as well as FLcDNA of the pig NPPB (see Figure 2a) were RT-PCR cloned into the BamHI and NcoI sites of the pCAL-n-FLAG expression vector (Stratagene, Madrid, Spain) and expressed in BL21 E. coli cells (Stratagene). Expressed proteins that fused to the 5-kDa tag, which included the calmodulinbinding peptide (CBP) and FLAG peptide, were purified from bacterial extracts, using calmodulinaffinity resin (Stratagene). Affinity-purified fusion proteins (with or without $10 \mathrm{mmol} / \mathrm{L}$ dithiothreitol [DTT]) were separated by sodium dodecyl sulfatepolyacrylamide gel electrophoresis (SDS-PAGE) followed by Western blot with anti-FLAG (Sigma) or with our-lab-derived anti-PLP antibodies (see below).

\section{COS-7 cell culture, transfection and transient expression assays}

COS-7 cells were cultured in Dulbecco's modified Eagle's Medium (Gibco, Barcelona, Spain) supplemented with $10 \%$ fetal bovine serum and penicillin-streptomycin-glutamine (Gibco) under standard tissue culture conditions at $37^{\circ} \mathrm{C}$. Cells were trypsinized at $70-80 \%$ confluence, cell numbers were determined using an automated cell counter (Countess, Invitrogen) and 80,000 cells were plated in each well of 12 -well plates and allowed to attach overnight. The $\triangle \mathrm{E} 2-\mathrm{NPPB}$ and NPPB variants, as well as the FL- $\triangle$ NKRD1 (ankyrin repeat domain 1 protein) were amplified from piglet LV oligo-dT-primed cDNA, cloned into pcDNA3.1/ myc-His B (Invitrogen) or p3XFLAG-CMV-14 (Sigma) vectors and verified by sequencing. Transfection of each CMVdriven plasmid expressing pig $\triangle \mathrm{E} 2-\mathrm{NPPB}, \mathrm{NPPB}$, ANKRD1 or control empty vector (see Figure 2a) was carried out with Lipofectamine LTX and PLUS Reagents (Invitrogen) following the manufacturer's instructions. For each plasmid or combination of plasmids, at least three separate transfection assays were employed, and in each assay, transfections were performed in duplicate. In some experiments, NPPB-transfected cells were cultivated in the presence of synthetic peptides comprising FL or partial amino acid (aa) sequences of the pig DE2-NPPB. The FL-DE2-NPPB peptide (aa 1-86) was synthesized by GeneScript (Piscataway, NJ, USA), while peptides labeled as KSW (aa 48-61) and PLP (aa 61-72) were synthesized by Davids Biotechnologie (Regensburg, Germany) (see Figure 4d). Samples of the respective peptides were mixed with Transport Protein Delivery Reagent (Lonza) before adding final concentrations of 50 and $100 \mathrm{nmol} / \mathrm{L}$ to the media. Changes in the status of transfected cells, such as cell number, cell morphology and cell adhesion or cell viability, were monitored in realtime using a realtime cell analyzer (RTCA) SP Station (Roche, Madrid, Spain) placed inside a tissue culture incubator, in accordance with the 
manufacturer's recommendations. In all transfection assays, after 48 hours in culture, the medium was saved (without disrupting the cells) and 10- to 30-fold concentrated (Amicon YM 3-kDa cutoff units). To obtain total protein lysates, the cells were rinsed with PBS, scraped into $2 \mathrm{x}$ Laemmli sample buffer (Invitrogen) and centrifuged $\left(20,000 \mathrm{~g}, 30 \mathrm{~min}, 15^{\circ} \mathrm{C}\right)$ saving the supernatants.

\section{SDS-PAGE and Western blotting}

Samples in a standard Laemmli sample buffer (Invitrogen) supplemented with complete protease inhibitors (Roche) were subjected to SDS-PAGE (Mini-Protean III, Bio-Rad), stained with Coomassie or blotted onto polyvinylidene fluoride membranes (Hybond-P, Amersham Biosciences, Barcelona, Spain) and probed with mouse monoclonal anti-myc and anti-FLAG antibodies (Sigma) or polyclonal immuno-affinity-purified anti-PLP antibodies. The antiserum against the PLP synthetic peptide, compromising aa residues $61-72$ of the pig $\triangle E 2-N P P B$ (see Figure 1d), was generated in rabbits by Davids Biotechnologies. Blocking, washing, incubation with diluted primary and secondary horse radish peroxidase-conjugated antibodies (Sigma) and visualization of immunodecorated bands by the Super-Signal West Pico chemiluminescent substrate (Pierce Biotechnology, Madrid, Spain) was carried out as described. ${ }^{19,21}$ Molecular weight (MW) standards (MARK-12 and SeeBlue Plus2 from Invitrogen) were included on each gel.

\section{Co-immunoprecipitation}

COS-7 cells were co-transfected with myc-tagged FL-NPPB and FLAG-tagged FL- $\Delta$ E2-NPPB After $48 \mathrm{~h}$ in culture, the cells were homogenized in lysis buffer $(50 \mathrm{mmol} / \mathrm{L}$ Tris, $\mathrm{pH} 8.0,200$ $\mathrm{mmol} / \mathrm{L} \mathrm{NaCl}, 1 \mathrm{mmol} / \mathrm{L}$ EDTA, $1 \%$ Triton X-100) supplemented by protease inhibitor cocktail (Roche). Lysates were centrifuged at $20,000 \mathrm{~g}$ for $20 \mathrm{~min}$, and supernatants were incubated overnight with FLAG(M2)-epitope antibodies coupled to agarose beads (Sigma). Beads were washed five times with lysis buffer, and bead-bound proteins were eluted by competition with 3xFLAG peptide (Sigma), resolved by SDS-PAGE, and Western blot probed with mouse monoclonal anti-myc or anti-FLAG antibodies (Sigma).

\section{Statistics}

Results are expressed as mean \pm standard error of the mean. Statistical significance was evaluated by Student's t-test. Statistical analyses were performed with SPSS 13 software. A value of $P \leq 0.05$ was considered statistically significant. 


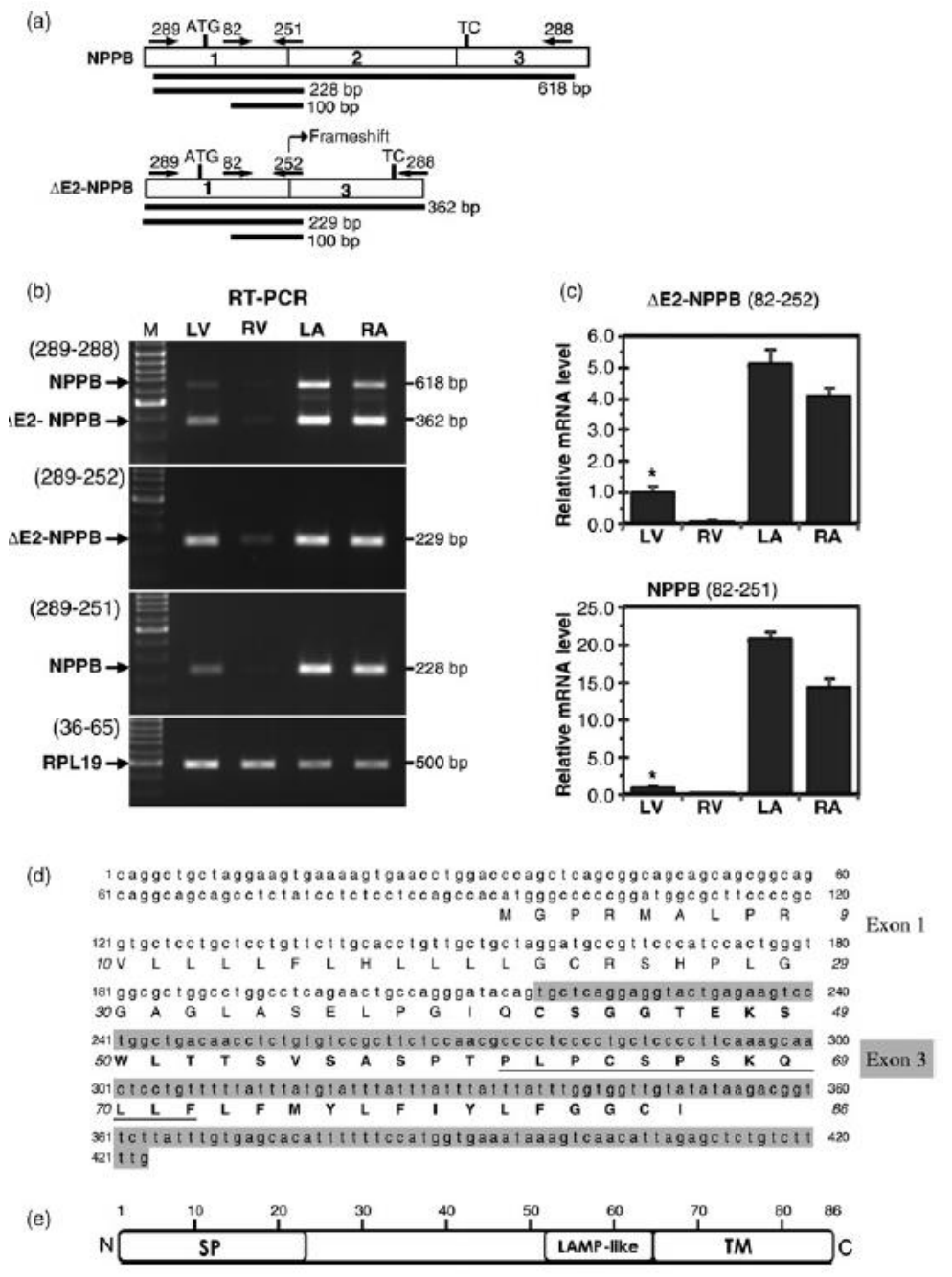

Figure 1 Identification, characterization and expression of a novel exon 2-skipped BNP variant ( $\triangle \mathrm{E} 2-\mathrm{NPPB})$ in newborn piglet heart. (a) Schematic representations of pig normally spliced NPPB (acc. no. NM_213846) and exon-skipped DE2NPPB sequences used for primer design: 1-3, exons; ATG, start codon; and TC, termination codon. The approximate location of the primers for downstream RT-PCR and qPCR analyses is indicated. The expected size of the corresponding amplicons is shown in black lines. ( $\mathrm{b}$ and c) $\triangle \mathrm{E} 2$-NPPB and NPPB mRNA expression in four cardiac chambers of newborn piglets as revealed by RT-PCR and qPCR, respectively (primers used are indicated in brackets). $* P \leq 0.05$. (d) Nucleotide and amino acid (capital letters) sequences of $\triangle \mathrm{E} 2$-NPPB. The exon 3 coding sequence is marked in gray. The amino acid sequence that was used to generate anti-PLP antibodies is underlined. (e) Domain motifs identified in the primary structure of the $\triangle \mathrm{E} 2-\mathrm{NPPB}$ protein. LAMP-like and TM domains were mapped using $\mathrm{CDD}^{29}$ and ConPred $\mathrm{II}^{30}$ algorithms, respectively. $\triangle \mathrm{E} 2-\mathrm{NPPB}$, alternatively spliced variant of porcine and human NPPB resulting from exon 2 skipping; NPPB, natriuretic peptide precursor B; qPCR, quantitative realtime RT-PCR; RT-PCR, reverse transcription-polymerase chain reaction; LV/RV, left/right ventricle; LA/RA, left/right atrium; LAMP, lysosome-associated membrane proteins; TM, transmembrane; SP, signal peptide 

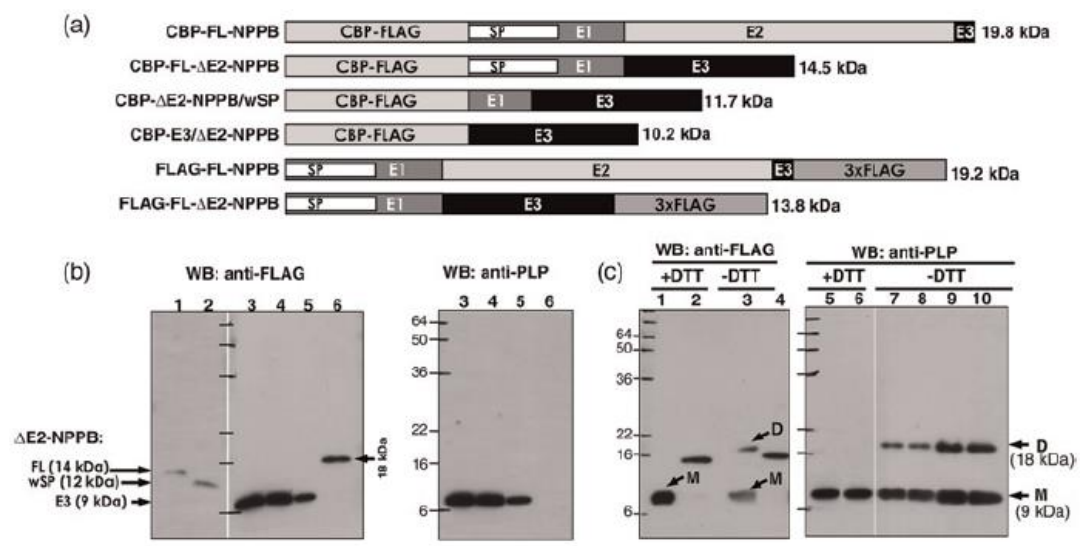

(d)
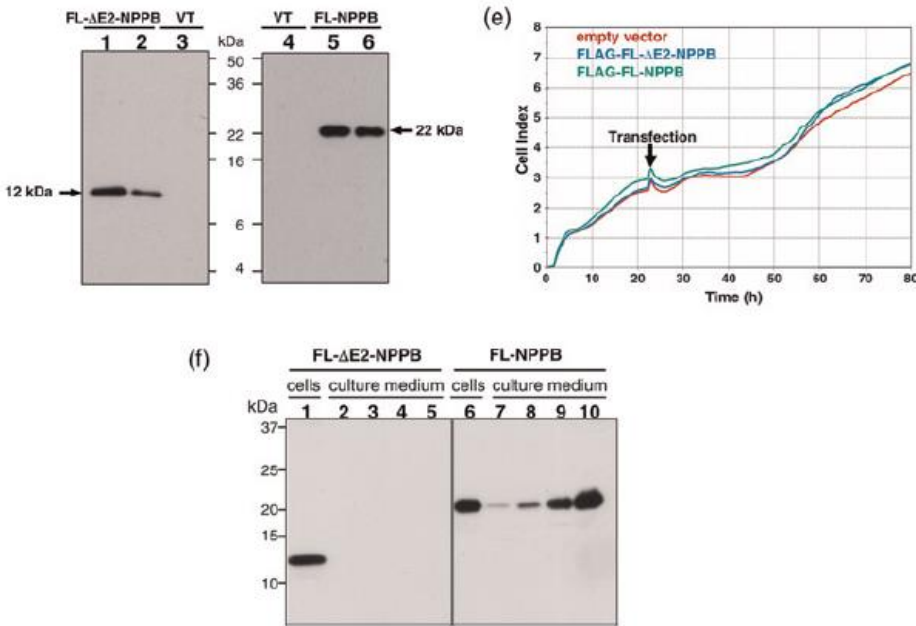

Figure 2 Protein expression analysis of the pig $\triangle E 2-N P P B$ in cell-based assays. (a) Schematic representation of CBPFLAG-tagged constructs carrying various configurations of pig NPPB and $\triangle E 2-N P P B$. Exon 3 (E3) coded and SP sequences are denoted with black and white boxes, respectively. Predicted MW values (kDa) are indicated. (b) Western blot (WB) detection of $E$. coli-expressed constructs with anti-FLAG (left panel) or anti-PLP (right panel) antibodies. Expressed constructs: FL- $\triangle$ E2-NPPB (lane 1), FL- $\triangle$ E2-NPPB/wSP (lane 2), E3/AE2-NPPB (lanes 3-5) and FL-NPPB (lane 6). MW values are indicated $(\mathrm{kDa})$. (c) Protein coded by E3 of the pig $\Delta \mathrm{E} 2-\mathrm{NPPB}(\mathrm{E} 3 / \triangle \mathrm{E} 2-\mathrm{NPPB}$ in a) was electrophoresed in the presence (+DTT, lanes 1, 5 and 6) or absence (-DTT, lanes 3, 7-10) of reducing agents and immunoblotted with anti-FLAG (left panel, lanes 1 and 3: E3/AE2-NPPB; lanes 2 and 4: NPPB) or anti-PLP (right panel, lanes 5-10: E3/ $\triangle E 2-N P P B$ ) antibodies. (d) COS-7 cells transfected with FLAG-tagged FL- $\triangle E 2-N P P B$ (left panel; lanes 1 and 2) or FL-NPPB (right panel; lanes 5 and 6) constructs were immunoblotted with anti-FLAG antibody. VT, vector-only transfected cells (lanes 3 and 4). (e) RTCA SP data plot of realtime scanning of COS-7 cell index (i.e. cell number, attachment and viability) before, at the moment and after transfections. (f ) COS-7 cells were transfected with FLAGtagged FL- $\triangle$ E2-NPPB (left panel) or FL-NPPB (right panel) constructs, and medium-free cell extracts (lanes 1 and 6 ) and cell-free unconcentrated (lanes 2 and 7) and two-fold (lanes 3 and 8), four-fold (lanes 4 and 9) and 12-fold (lines 5 and 10) concentrated culture medium samples were immunoblotted with anti-FLAG antibody. MW value, kDa. CBP, calmodulinbinding peptide; $\triangle \mathrm{E} 2-\mathrm{NPPB}$, alternatively spliced variant of porcine and human NPPB resulting from exon 2 skipping; NPPB, natriuretic peptide precursor B; SP, signal peptide; Wsp, without signal peptide; MW, molecular weight; DTT, dithiothreitol; M, monomers; D, dimmers; RTCA, realtime cell analyzer 


\section{Results}

Exon skipping generates a novel NPPB variant which is asymmetrically expressed in newborn piglet heart

Two bands were detected by RT-PCR analysis of the NPPB transcript (exon 1/primer 289exon 3/primer 288; Figure 1a) in each chamber of the newborn piglet heart (Figure 1b). Sequence analysis of the bands revealed that the 618-bp band carried an entire sequence of exons 1 and 2 , and approximately $80 \%$ of exon 3 of the $N P P B$ gene, while the 362-bp band contained exons 1 and 3 , but skipped exon 2 (Figure 1d). The complete cDNA of this NPPB variant, including the signal peptide sequence, was designated as $\triangle \mathrm{E} 2-\mathrm{NPPB}$. Both the $\triangle \mathrm{E} 2-\mathrm{NPPB}$ and normally spliced NPPB transcript showed a similar asymmetric distribution in the newborn heart, being more abundant in atria than in ventricles and in left ventricle/ atrium as compared with right ventricle/atrium (Figure $1 \mathrm{c})$.

Figure 1d shows nucleotide and aa sequences of the $\triangle \mathrm{E} 2-\mathrm{NPPB}$ variant. Skipping of exon 2 causes a frameshift in the open-reading frame (from the beginning of exon 3 ) generating a coding sequence, which would be expected to produce a novel 86 aa peptide (with theoretical pI/MW: 8.9/9.2) that is unique and distinct from other known protein families. Bioinformatic analysis of the $\triangle \mathrm{E} 2-\mathrm{NPPB}$ aa sequence revealed the following structural features (Figure 1e): (1) a signal peptide (aa 1-23); (2) a putative domain (aa 52-66) resembling the Lamp family of lysosomeassociated membrane proteins (i.e. LAMP-like domain); and (3) a transmembrane domain (aa 6585 ). The C-terminal halfsequence of $\triangle \mathrm{E} 2-\mathrm{NPPB}$ also contains three cysteine residues and is likely to be able to form internal di-sulfide bonds required for a classical 'LAMP fold'. ${ }^{22}$

Exon-skipped NPPB transcript is effectively translated into protein in cell-based expression systems

The coding region of the FL sequence, with and without signal peptide, and the exon 3 coding sequence of pig $\triangle \mathrm{E} 2-\mathrm{NPPB}$, as well as the coding sequence of pig FL-NPPB (with signal peptide) were cloned into the prokaryotic pCAL-n-FLAG vector and overexpressed in E. coli (Figure 2a). The affinity-purified single proteins were probed by SDS-PAGE followed by Western blot with anti-FLAG or with our lab-derived anti-exon 3- $\triangle \mathrm{E} 2-\mathrm{NPPB}$ antibodies (named anti-PLP) (Figure $2 \mathrm{~b}$ ). The anti-PLP antibodies raised against the synthetic peptide located in the pig $\triangle \mathrm{E} 2-\mathrm{NPPB} \mathrm{CT}$ region recognized its exon 3-coded peptide, but did not recognize either FL- $\Delta E 2-N P P B$ or FLNPPB (Figure 2b). Based on immunoblot analysis, we observed that the mobility of the expressed constructs is consistent with their predicted MW. In addition, the results revealed that elevated production of the $\triangle E 2-N P P B$ exon 3-coded peptide in $E$. coli could result in its dimerization. Coelectrophoresis of the DTT-free and DTT-treated samples demonstrated that this peptide does form dimers in the absence of a reducing agent (Figure $2 c$ ).

To further confirm that the $\triangle \mathrm{E} 2-\mathrm{NPPB}$ could be effectively translated in vivo, we constructed a CMV promoter-driven FL- $\triangle \mathrm{E} 2-\mathrm{NPPB}$ cDNA expression vector to enable its functional characterization in the mammalian COS-7 cell transfection system. The FLAG-tagged FL-NPPB expression vector was used in parallel as a reference. Transient expression in COS-7 cells demonstrated that the FL- $\triangle \mathrm{E} 2-\mathrm{NPPB}$ variant translated to a stable peptide able to accumulate to readily detectable levels within transfected cells (Figure 2d). $\triangle \mathrm{E} 2-\mathrm{NPPB}$-transfected COS-7 cells displayed no apparent difference in viability, replication rate or phenotype compared with FLNPPB- and mocktransfected cells (see Figure 2e). Similar levels of FL- $\triangle$ E2-NPPB and FL-NPPB were detected in COS-7 cell extracts; however, only FL-NPPB was detected in conditioned medium (Figure 2f ). These results indicated that differences in the secretion properties of FL$\triangle \mathrm{E} 2-\mathrm{NPPB}$ and FL-NPPB are not related to the signal peptide, which they have in common. 
Expression of exon-skipped NPPB is downregulated in the piglet myocardium during early postnatal development

Cardiac $\triangle \mathrm{E} 2$-NPPB mRNA levels were reduced in 30-day-old compared with newborn animals, but the extent of the decrease was different in each heart chamber (Figure 3a). The maximum reduction in $\triangle \mathrm{E} 2-\mathrm{NPPB} \mathrm{mRNA}$ levels was observed in the RV (21.6-fold change), together with its lesser but significant downregulation in the LV (12.7-fold change) of 30-day-old animals. In contrast, in the right and left atria of these piglets, decreased $\triangle \mathrm{E} 2-\mathrm{NPPB}$ gene expression was significantly less (5.6- and 1.7-fold change, respectively) in comparison with the changes observed in the ventricles.

Expression of exon-skipped NPPB is markedly re-activated in piglet model of diastolic heart failure

NPPB levels are predictive of impaired diastolic function. ${ }^{6,7}$ In this work, experimental diastolic heart failure (DHF) was induced in six-day-old piglets by a single Dox injection. At day 30, neonatal piglets developed a severe biventricular diastolic dysfunction resulting in pulmonary congestion. ${ }^{19}$ We quantified (by qPCR) the relative amounts of the $\triangle \mathrm{E} 2-\mathrm{NPPB}$ splice variant in the LV/RV myocardium of control (i.e. PBS-injected) and experimental (i.e. Dox-injected) piglets (Figures $3 \mathrm{~b}$ and $\mathrm{c}$ ). In 30-day-old piglets with Dox-induced DHF, the $\triangle \mathrm{E} 2-\mathrm{NPPB}$ mRNA levels were consistently and significantly augmented in both ventricles; $\triangle \mathrm{E} 2-\mathrm{NPPB}$ mRNA levels were more upregulated in the RV (38.8-fold change) than in the LV (9.4-fold change) as compared with age-matched PBS-injected controls. The average fold-increase of the normally spliced NPPB mRNA in the failing versus control RV and LV myocardium was only 6.3 and 5.1, respectively (data not shown).

Exon-skipped NPPB attenuates accumulation and secretion of normally spliced NPPB in cotransfection assays

Because $\triangle \mathrm{E} 2-\mathrm{NPPB}$ and normally spliced NPPB variants are co-expressed in the normal piglet myocardium and co-upregulated in the failing piglet myocardium, we were interested in determining if the presence of a non-secreted $\triangle \mathrm{E} 2-\mathrm{NPPB}$ isoform might influence rates of accumulation/ secretion of the protein isoform produced by the normally spliced NPPB transcript. In order to test this possibility, we simultaneously co-transfected COS-7 cells with: (1) a constant concentration of a plasmid encoding FLAG-tagged FL-NPPB; (2) ascending concentrations of a plasmid encoding myc-tagged FL- $\triangle \mathrm{E} 2-\mathrm{NPPB}$; and (3) descending concentrations of empty-vector to normalize total plasmid DNA concentration in different co-transfections. COS-7 cells transfected with the different plasmids were then harvested for Western blot and RT-PCR analyses. Figure $4 \mathrm{a}$ shows the detection of NPPB and $\triangle \mathrm{E} 2-\mathrm{NPPB}$ protein expression. However, the most striking observation was the dramatic inhibition of NPPB accumulation (Figure 4a, upper panel, lanes 4-6) when co-expressed with increasing amounts of the $\triangle E 2-N P P B-e x p r e s s i n g$ plasmid, but not with empty vector alone (Figure 4a, upper panel, lane 1) or control non-relevant protein (Figure 4c, upper panel, lanes 2-6), where similar amounts of the NPPB protein isoform were detected. Of note, inhibition of NPPB accumulation in transfected cells was associated with its reduced secretion into the media (Figure $4 \mathrm{a}$, lower panels). Figure $4 \mathrm{~b}$ shows the overall results of four independent repetitions of this setup assay demonstrating a dosedependent inhibition of NPPB accumulation by $\triangle \mathrm{E} 2-\mathrm{NPPB}$ in co-transfected COS-7 cells.

To exclude possible non-specific side-effects of elevated $\Delta \mathrm{E} 2$-NPPB protein concentrations on NPPB translation per se, we performed additional co-transfection experiments using the NPPB vector in combination with non-relevant plasmid expressing the pig ANKRD1. The levels of myctagged NPPB protein were unchanged in spite of extremely high levels of FLAG-tagged ANKRD1 accumulation in co-transfected COS-7 cells (Figure 4c, compare lanes 1-3 with lanes 4-6), respectively. In DE2-NPPB/NPPB co-transfections (Figure 4b), the NPPB mRNA levels (determined by RT-PCR) were not affected, ruling out a possible effect of overexpressed DE2NPPB on the CMV promoter function. Co-immunoprecipitation failed to detect endogenous 
interaction between $\triangle \mathrm{E} 2-\mathrm{NPPB}$ and NPPB isoforms co-expressed in COS-7 cells (data not shown).

To determine whether exogenously added $\triangle \mathrm{E} 2-\mathrm{NPPB}$ protein could also influence NPPB accumulation, we monitored the NPPB expression levels (determined by Western blot) in transfected COS-7 cells cultivated in the presence of the synthetic FL- $\triangle E 2-N P P B$ or its CTderived peptides in the media (Figure 4d), respectively. At the concentrations used in this assay, all synthetic peptides inhibited NPPB protein accumulation in transfected cells, with the inhibitory action of the KSW peptide most evident (Figure 4e and f ). Of note, the extent of the inhibitory effect of the KSW peptide was comparable with that of the synthetic FL- $\Delta$ E2-NPPB. This suggests that the inhibitory activities of $\triangle \mathrm{E} 2-\mathrm{NPPB}$ on the post-transcriptional expression of normally spliced NPPB are associated with sequence elements residing in exon 3 of $\triangle \mathrm{E} 2-\mathrm{NPPB}$.

(a)
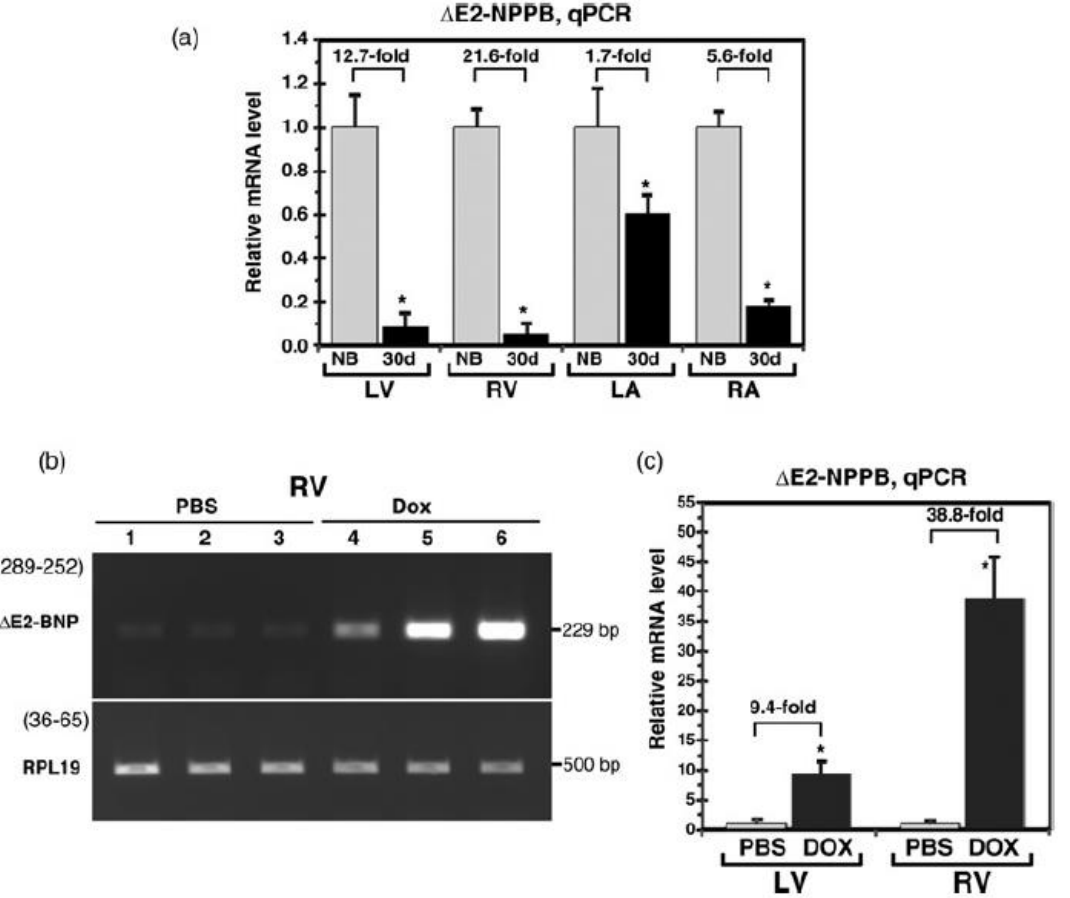

Figure 3 Cardiac $\triangle \mathrm{E} 2-\mathrm{NPPB} \mathrm{mRNA}$ levels in piglet postnatal development and diastolic heart failure. (a) Average $\triangle \mathrm{E} 2-$ NPPB mRNA levels in cardiac chambers of newborn $(\mathrm{NB}, \mathrm{n}=6$ ) versus 30 -day-old (30d, $n=6)$ piglets. $\mathrm{P} \leq 0.05$. (b) Representative semiquantitative RT-PCR for $\triangle \mathrm{E} 2-\mathrm{NPPB}$ (target) and RPL19 (reference) transcripts in the RV myocardium of Dox- $(n=3)$ versus PBS-injected $(n=3)$ 30d piglets. Primer pairs used are indicated in brackets. (c) Average fold increase in LV/RV $\triangle \mathrm{E} 2$-NPPB mRNA levels in Dox-injected $(n=12)$ versus PBS-injected $(n=12)$ piglets estimated by qPCR analysis. $\mathrm{P} \leq 0.05 . \triangle \mathrm{E} 2-\mathrm{NPPB}$, alternatively spliced variant of porcine and human NPPB resulting from exon 2 skipping; PBS, phosphate buffered saline; RT-PCR, reverse transcription-polymerase chain reaction; qPCR, quantitative realtime RT-PCR; RPL19, ribosomal protein L19; LV/RV, left/right ventricle; LA/RA, left/right atrium; Dox, doxorubicin

\section{Exon-skipped NPPB variants are detected in normal and failing human myocardium}

We first surveyed the presence of exon-skipped NPPB variants in cardiac samples from an eight-week-old patient. Two novel exon-skipped NPPB variants were readily detectable by our RT-PCR assay in human myocardium: one similar to the porcine $\triangle E 2-N P P B$ with exon 2 completely skipped (named $\triangle \mathrm{E} 2$ human NPPB or $\triangle \mathrm{E} 2-\mathrm{hNPPB}$ ) and the other transcript with a partially skipped exon 1 and completely skipped exon 2 named p $\Delta \mathrm{E} 1 / \Delta \mathrm{E} 2-\mathrm{hNPPB}$ (Figure 5a and b). According to sequence analyses, the $\triangle \mathrm{E} 2-\mathrm{hNPPB}$ is an ortholog of the porcine $\triangle \mathrm{E} 2-\mathrm{NPPB}$ variant, while the $\mathrm{p} \triangle \mathrm{E} 1 / \triangle \mathrm{E} 2-\mathrm{hNPPB}$ represents a transcript without any bioinformatics-predicted 
open-reading frame. We also compared exon-skipped NPPB expression in RNA samples from non-failing and failing human myocardium (Figure 5c). The level of expression of the two exonskipped NPPB transcripts is significantly upregulated in LV tissue samples from explanted as compared with those from donor human hearts (Figure 5d).

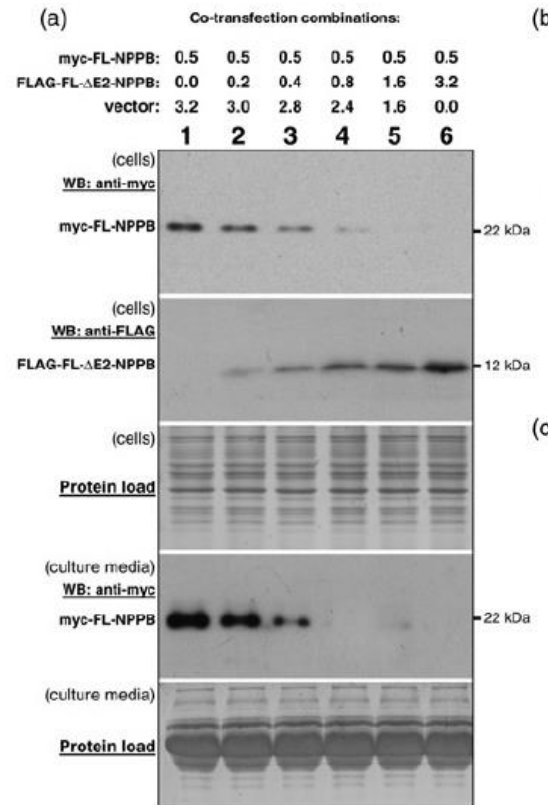

(b)

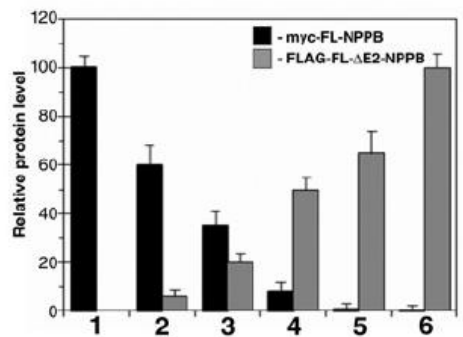

(c)

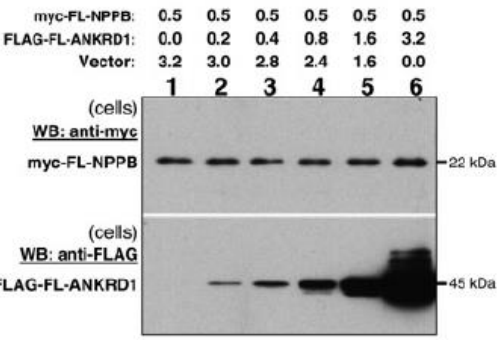

(d)
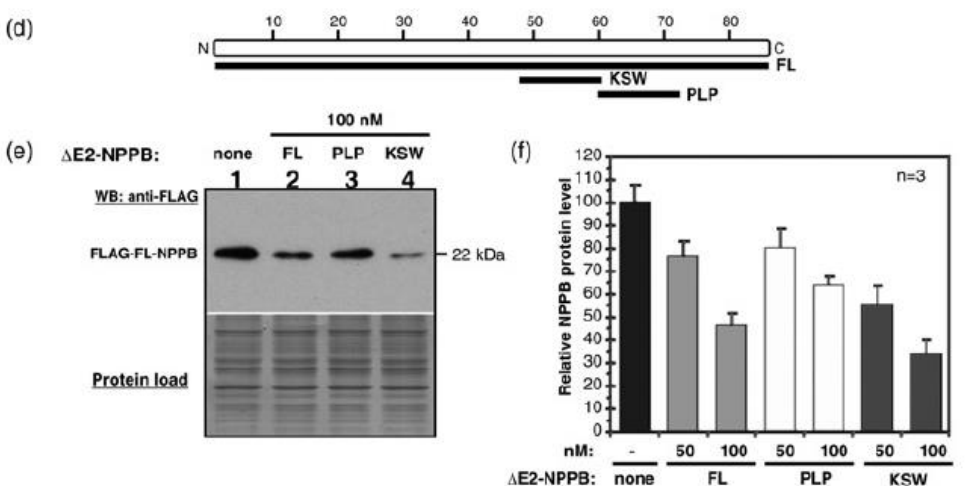

Figure 4 Relevance of the $\triangle \mathrm{E} 2-\mathrm{NPPB}$ protein to production and secretion of normally spliced NPPB precursor as revealed by co-transfection cell assays. (a) Total cell protein lysates (cells) and cell-free media (culture medium) from COS-7 cells co-transfected with $m y c$-tagged FL-NPPB, FLAG-tagged FL- $\triangle E 2-N P P B$ and empty vector were electrophoresed and immunoblotted (WB) with anti-myc or anti-FLAG antibodies. MW values (kDa) of the bands detected are shown. (b) Inverse relationship between NPPB and $\triangle \mathrm{E} 2-\mathrm{NPPB}$ protein expression in co-transfected COS-7 cells. Data from four replicates of each co-transfection combinations (lanes 1-6 as indicated in a) were pooled and averaged. (c) Total cell protein lysates (cells) of COS-7 cells co-transfected with myc-tagged FL-NPPB, FLAG-tagged FL-ANKRD1 (non-relevant protein expression control) and empty vector were electrophoresed and immunoblotted (WB) with anti-myc or anti-FLAG antibodies. MW values $(\mathrm{kDa})$ of the bands detected are shown. (d) Schematic mapping of synthetic FL, KSW and PLP peptides (black solid lines) to the complete $\triangle \mathrm{E} 2-\mathrm{PNNB}$ protein sequence. (e) COS-7 cells transfected with FLAG-tagged FL-NPPB were cultivated in the medium complemented with: protein-transfection vehicle (lane 1), FL- $\Delta$ E2-NPPB (lane 2), PLP (lane 3) and KSW (lane 4) synthetic peptides. The whole protein cell lysates were immunoblotted (WB) with antiFLAG antibody. MW values (kDa) of the bands detected are shown. (f ) Average inhibition effects of FL-, PLP- and KSW$\triangle E 2-N P P B$ synthetic peptides on NPPB protein accumulation into transfected COS-7 cells, respectively. $\triangle E 2-N P P B$, alternatively spliced variant of porcine and human NPPB resulting from exon 2 skipping; NPPB, natriuretic peptide precursor B; FL, full length; ANKRD1, ankyrin repeat domain 1 protein; MW, molecular weight 

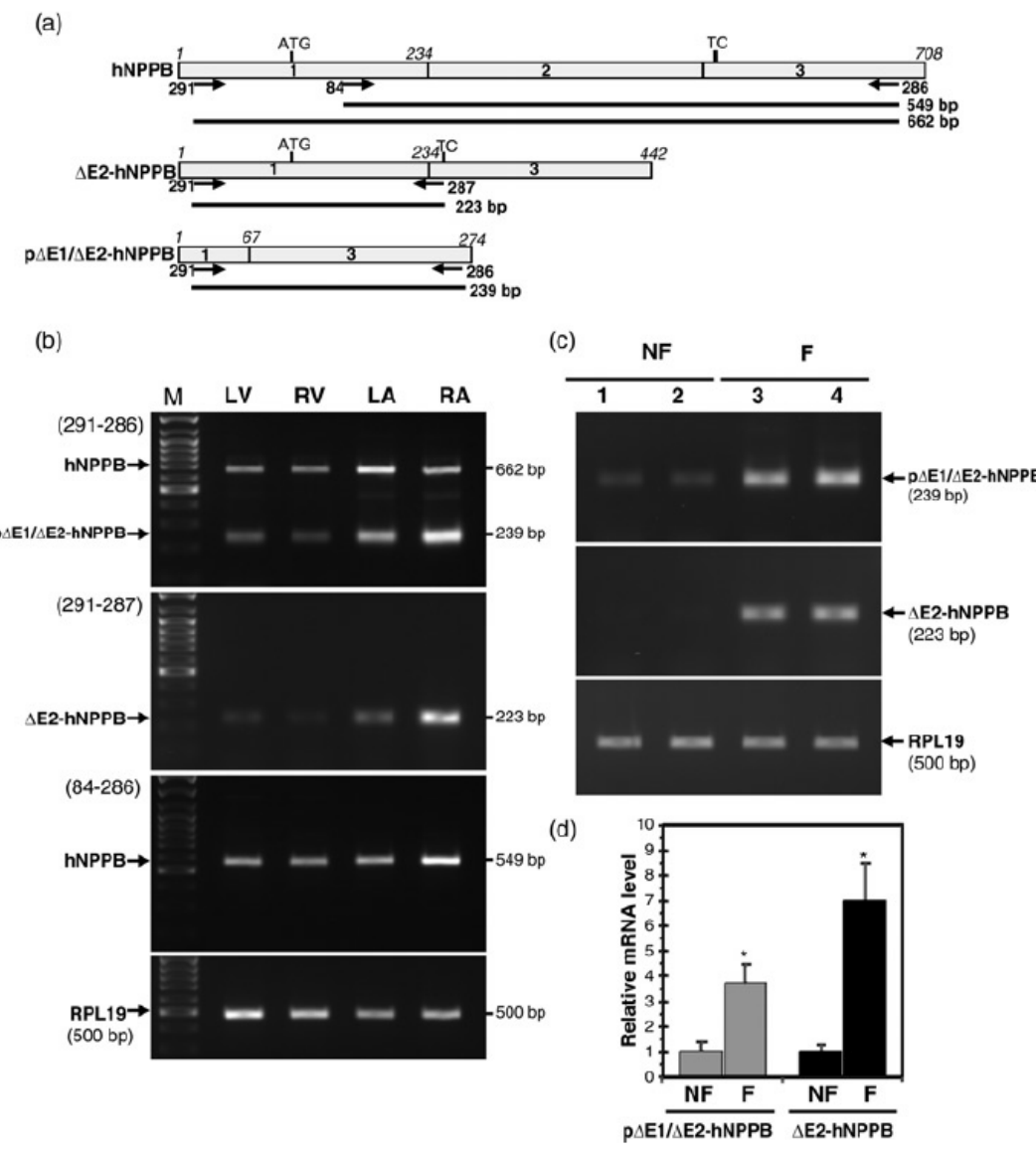

Figure 5 Identification and expression profiling of exon-skipped variants of NPPB in human myocardium. (a) Schematic representations of human (h) normally spliced NPPB (acc. no.: NM_002521) and exon-skipped NPPB sequences used for primer design: 1-3, exons; ATG and TC, start and termination codon, respectively. The approximate location of the primers for downstream RT-PCR analysis is indicated. (b) In addition to the normally spliced hNPPB transcript, exonskipped NPPB variants $(\mathrm{p} \triangle \mathrm{E} 1 / \triangle \mathrm{E} 2-\mathrm{hNPPB}$ and $\triangle \mathrm{E} 2-\mathrm{hNPPB})$ are detected in myocardium of an eight-week-old patient as revealed by semiquantitative RT-PCR. Primers 291-286 co-amplified two RT-PCR bands corresponding to hNPPB and $\mathrm{p} \triangle \mathrm{E} 1 / \triangle \mathrm{E} 2-\mathrm{hNPPB}$, whereas primers 291-287 and 84-286 selectively amplified $\triangle \mathrm{E} 2-\mathrm{hNPPB}$ and hNPPB, respectively. The expected size of the corresponding amplicons is shown in black lines. (c) Representative semiquantitative RT-PCR for target (p $\triangle \mathrm{E} 1 / \triangle \mathrm{E} 2-\mathrm{hNPPB}$ and $\triangle \mathrm{E} 2-\mathrm{hNPPB})$ and reference (RPL19) transcripts in NF versus F LV samples. Primer pairs used are indicated in brackets. The size of the corresponding amplicons is shown. (d) Average $\mathrm{p} \Delta \mathrm{E} 1 / \triangle \mathrm{E} 2-\mathrm{hNPPB}$ and $\triangle \mathrm{E} 2-$ hNPPB mRNA levels in NF $(n=5)$ versus F $(n=5)$ LV samples. $* P \leq 0.05$. NPPB, natriuretic peptide precursor B; RTPCR, reverse transcription-polymerase chain reaction; RPL19, ribosomal protein L19; NF, non-failing; F, failing; LV, left ventricle

\section{Discussion}

In the present study, we report a novel variant of the NPPB mRNA, ubiquitously expressed in pig cardiac tissues, which, if translation does occur, would generate a novel protein isoform that terminates 40 aa downstream of the correctly terminated NPPB product (see Figure 1a and e). This means that this variant would not proceed to form a classical 'ring' structure characteristic of mature NPPB. Further, exon 2 deletion causes a frameshift in the open-reading frame generating a coding sequence which would be expected to produce a previously unrecognized $\triangle \mathrm{E} 2-\mathrm{NPPB}$ isoform with homology restricted to only the exon 1 coding sequence of normally spliced pig NPPB. Our cell-based translation assays and immunoblot analysis revealed that FL- $\triangle \mathrm{E} 2-\mathrm{NPPB}$ is expressed with no significant differences in expression level compared with that of the normally spliced NPPB. However, the $\triangle \mathrm{E} 2-\mathrm{NPPB}$ protein, unlike the NPPB, was detected only in the 
cytosol of transfected cells, indicating that the deletion of exon 2 impairs its secretion into the culture media.

We found that both $\triangle \mathrm{E} 2-\mathrm{NPPB}$ and normally spliced NPPB are co-expressed in normal piglet myocardium and co-upregulated in failing piglet myocardium (see Figure 3 ). The next question we addressed was whether the co-regulated expression of two NPPB variants in normal and failing myocardium, one secreted and the other not, is of any functional significance. To answer this question, we simultaneously co-transfected COS-7 cells with alternatively spliced pig $\triangle \mathrm{E} 2-\mathrm{NPPB}$ and normally spliced NPPB. Under these experimental conditions, NPPB protein levels were reduced in inverse proportion to the increased amount of synthesized DE2-NPPB (see Figure 4b), indicating that $\triangle \mathrm{E} 2-\mathrm{NPPB}-$ mediated inhibition of NPPB protein accumulation/ secretion is dose dependent. Such inhibitory effects were mimicked by introducing the synthetic DE2-NPPB or its CT-derived peptides (KSW peptide) into the media of COS-7 cells transfected with only NPPB plasmid. It should be stressed that inhibition of NPPB protein accumulation/ secretion by $\triangle \mathrm{E} 2-$ NPPB did not affect the levels of normally spliced NPPB mRNA in co-transfected COS-7 cells. Thus, the decreased translational yield of NPPB could either be due to the decreased translatability of the normally spliced NPPB message or to the diminished stability of the encoded polypeptide. In this regard, we observed that the downregulation of NPPB protein levels by $\triangle \mathrm{E} 2-\mathrm{NPPB}$ could be partially reversed (up to $20-40 \%$ ) by cultivation of the co-transfected COS-7 cells in the media supplemented with protease inhibitors such as leupeptin or chloroquine (data not shown).

Our data indicate that pig $\triangle \mathrm{E} 2-\mathrm{NPPB}$ and NPPB are proteins with distinct molecular properties and retention/ secretion dynamics. As such, one might expect that these isoforms encoded by the two splice NPPB variants would tend to operate independently, with non-complementary roles with regard to each other. ${ }^{15}$ Instead, our data from cell-based assays support a model in which $\triangle \mathrm{E} 2-\mathrm{NPPB}$ appears to attenuate production of normally spliced NPPB, suggesting a negative feedback loop of NPPB signaling through generation of $\triangle E 2-N P P B$. Given that these protein isoforms do not interact with each other in co-precipitation assays, it may be that $\triangle \mathrm{E} 2-\mathrm{NPPB}$ affects the rate of NPPB production/accumulation indirectly.

The post-transcriptional mechanisms, which control intracardiac production and secretion of $\mathrm{NPPB}$, are as yet poorly elucidated. Lowering of intracellular concentrations of calcium and calcium-dependent proteins, such as calcineurin ${ }^{23}$ and calcium-calmodulin kinase II $^{24}$ could result in inhibition of NPPB production/secretion. In addition, blocking membrane calcium channels significantly reduced NPPB secretion by both normal and hypertrophied cardiomyocytes. ${ }^{25}$ The present study suggests a new pathway that is mediated by the alternatively spliced $\triangle \mathrm{E} 2-\mathrm{NPPB}$ variant for control of NPPB expression and secretion. This raised the relevant clinical question of whether exon-skipped NPPB variants are expressed in failing human myocardium. Our results show that two exon-skipped NPPB transcript variants are detected in normal and upregulated in failing human myocardium. In human myocardium, NPPB exon-skipping results in the generation of one variant without a bioinformatics-predicted open-reading frame (i.e. $\mathrm{p} \Delta \mathrm{E} 1 / \triangle \mathrm{E} 2-\mathrm{hNPPB}$ ), while it is predicted that the other (i.e. $\triangle \mathrm{E} 2-\mathrm{hNPPB}$ ) variant will have a premature termination codon (see Figure 5). Such mRNAs might be sensitive to the nonsense-mediated mRNA decay (NMD) pathway. ${ }^{26}$ However, transcripts harboring premature termination codons can escape NMD, leading to the synthesis of truncated proteins that may exert a dominant negative effect. ${ }^{27}$ This interpretation is consistent with our findings, insofar as $\triangle \mathrm{E} 2-\mathrm{NPPB}$ does attenuate NPPB production and secretion in cell-based co-transfection assays.

In summary, we provide here three novel observations relevant to the regulation of cardiac expression of the NPPB gene under physiological and pathological conditions: (1) normal pig and human myocardium exhibit alternative splicing of the NPPB mRNA, based on exon skipping; (2) intracellular levels and secretion of normally spliced NPPB are negatively regulated by their exonskipped variants at a post-transcriptional level; and (3) exon-skipped NPPB variants are upregulated in failing myocardium in both the porcine model and patients with end-stage HF. Given that human HF is a state of deficiency of active NPPB, ${ }^{28}$ the demonstrated negative feedback relationship between $\triangle \mathrm{E} 2-\mathrm{NPPB}$ and NPPB expression may be especially relevant in further studying the molecular, functional and clinical consequences of exon-skipped NPPB variant expression in patients. 
Author contributions: All authors participated in the design, interpretation and analysis of the data and review of the manuscript; MT, RI and ATM conducted molecular, biochemical and cellbased experiments; AC and EL conducted experimental animal studies; and ATM and MT wrote the manuscript.

\section{ACKNOWLEDGEMENTS}

The authors thank Drs Marisa Crespo, Javier Muñiz and Constancio Medrano (University Hospital Centre of La Coruña, La Coruña, Spain) for their help in sampling and characterization of human heart tissues. Special thanks to Cristina Trillo (Roche, Spain) and Javier Aldama (Roche, Spain) for the opportunity to use the RTCA SP Station (Roche) in our cell-transfection experiments. This work was supported by grants (SAF2004-01462 and SAF2008- 00337) from the Spanish Ministry of Science and Innovation and partially by a grant (08CSA008161PR) from the Autonomic Government of Galicia.

\section{REFERENCES}

1. Vanderheyden M, Bartunek J, Goethals M. Brain and other natriuretic peptides: molecular aspects. Eur J Heart Fail 2004;6:261-8

2. Nakayama $T$. The genetic contribution of the natriuretic peptide system to cardiovascular diseases. Endocr J 2005;52:11-21

3. Burke MA, Cotts WG. Interpretation of B-type natriuretic peptide in cardiac disease and other comorbid conditions. Heart Fail Rev 2007;12:23-36

4. Zakynthinos E, Kiropoulos T, Gourgoulianis K, Filippatos G. Diagnostic and prognostic impact of brain natriuretic peptide in cardiac and noncardiac diseases. Heart Lung 2008;37:275-85

5. Iwanaga Y, Nishi I, Furuichi S, Noguchi T, Sase K, Kihara Y, Goto Y, Nonogi H. B-type natriuretic peptide strongly reflects diastolic wall stress in patients with chronic heart failure: comparison between systolic and diastolic heart failure. J Am Coll Cardiol 2006;47:742-8

6. Grewal J, McKelvie RS, Persson H, Tait P, Carlsson J, Swedberg K, Ostergren J, Lonn E. Usefulness of $\mathrm{N}$-terminal pro-brain natriuretic peptide and brain natriuretic peptide to predict cardiovascular outcomes in patients with heart failure and preserved left ventricular ejection fraction. Am J Cardiol 2008;102:7337

7. Grewal J, McKelvie R, Lonn E, Tait P, Carlsson J, Gianni M, Jarnert C, Persson H. BNP and NT-proBNP predict echocardiographic severity of diastolic dysfunction. Eur J Heart Fail 2008;10:252-9

8. LaPointe MC. Molecular regulation of the brain natriuretic peptide gene. Peptides 2005;26:944-56

9. Mair J. Biochemistry of B-type natriuretic peptide - where are we now? Clin Chem Lab Med 2008;46:1507-14

10. Vanderheyden M, Goethals M, Verstreken S, De Bruyne B, Muller K, Van Schuerbeeck E, Bartunek J. Wall stress modulates brain natriuretic peptide production in pressure overload cardiomyopathy. $\mathrm{J}$ Am Coll Cardiol 2004;44:2349-54

11. Frank D, Kuhn C, Brors B, Hanselmann C, Ludde M, Katus HA, Frey N. Gene expression pattern in biomechanically stretched cardiomyocytes: evidence for a stretch-specific gene program. Hypertension 2008;51:309-18

12. Majalahti T, Suo-Palosaari M, Sarman B, Hautala N, Pikkarainen S, Tokola H, Vuolteenaho O, Wang J, Paradis P, Nemer M, Ruskoaho H. Cardiac BNP gene activation by angiotensin II in vivo. Mol Cell Endocrinol 2007;273:59-67

13. Suo M, Hautala N, Foldes G, Szokodi I, Toth M, Leskinen H, Uusimaa P, Vuolteenaho O, Nemer M, Ruskoaho H. Posttranscriptional control of BNP gene expression in angiotensin II-induced hypertension. Hypertension 2002;39:803-8

14. Tenhunen O, Szokodi I, Ruskoaho H. Posttranscriptional activation of BNP gene expression in response to increased left ventricular wall stress: role of calcineurin and PKC. Regul Pept 2005;128:187-96

15. Pan S, Chen HH, Dickey DM, Boerrigter G, Lee C, Kleppe LS, Hall JL, Lerman A, Redfield MM, Potter LR, Burnett JC Jr, Simari RD. Biodesign of a renal-protective peptide based on alternative splicing of Btype natriuretic peptide. Proc Natl Acad Sci USA 2009;106:11282-7

16. Torrado M, Iglesias R, Nespereira B, Mikhailov AT. Identification of candidate genes potentially relevant to chamber-specific remodeling in postnatal ventricular myocardium. J Biomed Biotechnol 2010; doi: $10.1155 / 2010 / 603159$ 
17. Torrado M, Lopez E, Centeno A, Castro-Beiras A, Mikhailov AT. Left-right asymmetric ventricular expression of CARP in the piglet heart: regional response to experimental heart failure. Eur J Heart Fail 2004;6:161-72

18. Torrado M, Centeno C, Lo'pez E, Mikhailov AT. In-vivo forced expression of myocardin in ventricular myocardium transiently impairs systolic performance in early neonatal pig heart. Int J Dev Biol 2009;53:1457-67

19. Torrado M, Nespereira B, Bouzamayor Y, Centeno A, Lopez E, Mikhailov AT. Differential atrial versus ventricular ANKRD1 gene expression is oppositely regulated at diastolic heart failure. FEBS Lett 2006;580:4182-7

20. Torrado M, Lopez E, Centeno A, Medrano C, Castro-Beiras A, Mikhailov AT. Myocardin mRNA is augmented in the failing myocardium: expression profiling in the porcine model and human dilated cardiomyopathy. J Mol Med 2003;81:566-77

21. Torrado M, Iglesias R, Nespereira B, Centeno A, Lopez E, Mikhailov AT. Intron retention generates ANKRD1 splice variants that are co-regulated with the main transcript in normal and failing myocardium. Gene 2009;440:28-41

22. David A, Tiveron MC, Defays A, Beclin C, Camosseto V, Gatti E, Cremer H, Pierre P. BAD-LAMP defines a subset of early endocytic organelles in subpopulations of cortical projection neurons. J Cell Sci 2007; 120:353-65

23. Tavi P, Pikkarainen S, Ronkainen J, Niemela P, Ilves M, Weckstrom M, Vuolteenaho O, Bruton J, Westerblad H, Ruskoaho H. Pacing-induced calcineurin activation controls cardiac $\mathrm{Ca}^{2+}$ signalling and gene expression. J Physiol 2004;554:309-20

24. Ronkainen JJ, Vuolteenaho O, Tavi P. Calcium-calmodulin kinase II is the common factor in calciumdependent cardiac expression and secretion of A- and B-type natriuretic peptides. Endocrinology 2007;148:2815-20

25. Nader L, Smayra V, Jebara V, Bois P, Potreau D, Fares N. Brain natriuretic peptide secretion in adult rat heart muscle cells: the role of calcium channels. Arch Cardiovasc Dis 2008;101:459-63

26. McGlincy NJ, Smith CW. Alternative splicing resulting in nonsense-mediated mRNA decay: what is the meaning of nonsense? Trends Biochem Sci 2008;33:385-93

27. Bashyam MD. Nonsense-mediated decay: linking a basic cellular process to human disease. Expert Rev Mol Diagn 2009;9:299-303

28. Chen HH. Heart failure: a state of brain natriuretic peptide deficiency or resistance or both! J Am Coll Cardiol 2007;49:1089-91

29. Marchler-Bauer A, Anderson JB, Chitsaz F, Derbyshire MK, DeWeese-Scott C, Fong JH, Geer LY, Geer RC, Gonzales NR, Gwadz M, He S, Hurwitz DI, Jackson JD, Ke Z, Lanczycki CJ, Liebert CA, Liu C, Lu F, Lu S, Marchler GH, Mullokandov M, Song JS, Tasneem A, Thanki N, Yamashita RA, Zhang D, Zhang N, Bryant SH. CDD: specific functional annotation with the Conserved Domain Database. Nucleic Acids Res 2009;37:D205-10

30. Arai M, Mitsuke H, Ikeda M, Xia JX, Kikuchi T, Satake M, Shimizu T. ConPred II: a consensus prediction method for obtaining transmembrane topology models with high reliability. Nucleic Acids Res 2004;32:W390-3 